\title{
Ideal based trace graph of matrices
}

\author{
T. Tamizh Chelvam*(D), M. Sivagami (D) \\ Department of Mathematics, Manonmaniam Sundaranar University, Abishekapatti, Tirunelveli 627012, \\ Tamil Nadu, India.
}

\begin{abstract}
Let $R$ be a commutative ring and $M_{n}(R)$ be the set of all $n \times n$ matrices over $R$ where $n \geq 2$. The trace graph of the matrix ring $M_{n}(R)$ with respect to an ideal $I$ of $R$, denoted by $\Gamma_{I^{t}}\left(M_{n}(R)\right)$, is the simple undirected graph with vertex set $M_{n}(R) \backslash M_{n}(I)$ and two distinct vertices $A$ and $B$ are adjacent if and only if $\operatorname{Tr}(A B) \in I$. Here $\operatorname{Tr}(A)$ represents the trace of the matrix $A$. In this paper, we exhibit some properties and structure of $\Gamma_{I^{t}}\left(M_{n}(R)\right)$.
\end{abstract}

Mathematics Subject Classification (2010). 16S50, 05C25, 13A15, $05 \mathrm{C} 69$

Keywords. trace graph, matrix ring, ideal-based, clique number

\section{Introduction}

The concept of associating graphs to commutative rings was first introduced by Beck [3]. He introduced the concept of zero-divisor graph of a commutative ring $R$ as an undirected graph whose vertices are the elements of $R$ with two distinct vertices $x$ and $y$ joined by an edge if and only if $x y=0$. Later on, Anderson and Livingston [2] modified the definition with vertex set, the set of all nonzero zero divisors of $R$ and introduced the zero-divisor graph $\Gamma(R)$ corresponding to a commutative ring $R$. In [9], Redmond introduced the notion of the zero-divisor graph with respect to an ideal $I$ of a commutative ring $R$, denoted by $\Gamma_{I}(R)$, as the graph with vertex set $\{x \in R \backslash I: x y \in I$ for some $y \in R \backslash I\}$, and two distinct vertices $x$ and $y$ are adjacent if and only if $x y \in I$. The concept of trace graph of a matrix ring over a commutative ring was introduced by Almahdi, Louartiti, and Tamekkante [1]. Several authors have extensively studied about zero-divisor graph with respectxzs to an ideal. For example one may refer [8]. Let $R$ be a commutative ring and $n$ be a positive integer. Let $M_{n}(R)$ denote the set of all $n \times n$ matrices over $R, M_{n}(R)^{*}$ denotes the set of all $n \times n$ non-zero matrices over $R$ and let $\operatorname{Tr}(A)$ be the trace of the matrix $A \in M_{n}(R)$. The trace graph of the matrix ring $M_{n}(R)$, denoted by $\Gamma_{t}\left(M_{n}(R)\right)$, is the simple undirected graph with vertex set $\left\{A \in M_{n}(R)^{*}\right.$ : there exists $B \in M_{n}(R)^{*}$ such that $\operatorname{Tr}(A B)=0\}$ and two distinct vertices $A$ and $B$ are adjacent if and only if $\operatorname{Tr}(A B)=0$. Further study on the trace graph of matrices was done by authors [10].

In this paper, as a parallel approach of generalization of $\Gamma(R)$ to $\Gamma_{I}(R)$, we generalize the notion of the trace graph $\Gamma_{t}\left(M_{n}(R)\right)$ of a matrix ring $M_{n}(R)$ to the trace graph $\Gamma_{I^{t}}\left(M_{n}(R)\right)$ with respect to an ideal $I$ of $R$. Actually $\Gamma_{I^{t}}\left(M_{n}(R)\right)$ is the simple undirected

\footnotetext{
*Corresponding Author.

Email addresses: tamche59@gmail.com (T. Tamizh Chelvam), siva9212@gmail.com (M. Sivagami)

Received: 04.11.2018; Accepted: 04.02.2019
} 
graph with vertex set $M_{n}(R) \backslash M_{n}(I)$ and two distinct vertices $A$ and $B$ are adjacent if and only if $\operatorname{Tr}(A B) \in I$. Note that if $A \in M_{n}(I)$, then $\operatorname{Tr}(A B) \in I$ for every $B \in M_{n}(R)$. Due to this, matrices in $M_{n}(I)$ are not considered for the vertex set of $\Gamma_{I^{t}}\left(M_{n}(R)\right)$. As usual, $E_{i j}$ denotes the matrix whose $i j^{\text {th }}$ entry is 1 and 0 elsewhere. For a set $X,|X|$ denotes the cardinality of $X, X \backslash Y$ denotes the set of elements that belong to $X$ and not to set $Y$. For basic definitions on rings, one may refer [6] and for noncommutative rings see $[5,7]$.

Let $G$ be a graph. For distinct vertices $x$ and $y$ of $G$, let $d(x, y)$ be the length of the shortest path between $x$ and $y(d(x, y)=\infty$ if there is no such path). The diameter of $G$ is $\operatorname{diam}(G)=\sup \{d(x, y): x$ and $y$ are distinct vertices of $G\}$. The girth of $G$, denoted by $\operatorname{gr}(G)$, is defined as the length of the shortest cycle in $G(\operatorname{gr}(G)=\infty$ if $G$ contains no cycles). For a graph $G$ and a vertex $v \in V(G)$, the eccentricity $e(v)$ of $v$ is the maximum distance to any vertex in the graph, i.e., $e(v)=\max _{u \in V(G)}\{\mathrm{d}(v, u)\}$. The radius $\operatorname{rad}(G)$ of a $G$ is the minimum eccentricity among all vertices in $G$ and a vertex of $G$ is a central vertex if $e(v)=\operatorname{rad}(G) . G$ is self-centered if every vertex is in the center i.e., $e(v)=\operatorname{rad}(G)$ for every vertex $v \in V(G)$. A subset $\Omega$ of $V(G)$ is called a clique if the induced subgraph of $\Omega$ is complete. The order of the largest clique in $G$ is its clique number, which is denoted by $\omega(G)$. The chromatic number of a graph $G$, denoted by $\chi(G)$, is the smallest number of colors needed to color the vertices of $G$ so that no two adjacent vertices share the same color. An independent set or stable set is a set of vertices in a graph $G$ such that no two of them are adjacent. A maximum independent set is an independent set of largest possible size for the given graph $G$. This size is called the independence number of $G$ and denoted by $\alpha(G)$.

If the edges of $G$ are partitioned into subgraphs $H_{1}, \ldots, H_{k}, \ldots H_{n}$, then we write $G \cong$ $H_{1} \oplus \cdots \oplus H_{n}$, and if $H_{i} \cong H_{j}$ for all $1 \leq i, j \leq k$, then we write $G \cong k H \oplus H_{k+1} \oplus \cdots \oplus H_{n}$, where $H \cong H_{i},(1 \leq i \leq k)$. For general reference of graph theoretical terms and results, we refer [11].

Remark 1.1. Let $R$ be a commutative ring and $n$ be a positive integer.

1. The graph $\Gamma_{I^{t}}\left(M_{1}(R)\right)$ coincides with $\Gamma_{I}(R)$ (ideal based zero-divisor graph of the ring $R$ ).

2. If $I=(0)$, then $\Gamma_{I^{t}}\left(M_{n}(R)\right)=\Gamma_{t}\left(M_{n}(R)\right)$ for all $n \geq 1$.

3. If $I=R$, then $\Gamma_{I^{t}}\left(M_{n}(R)\right)$ is the null graph.

Throughout this paper, unless otherwise specified, $R$ is a commutative ring with identity, $n \geq 2$ is an integer, and $I$ is a non-trivial ideal of $R$. If $A=\left[a_{i j}\right] \in M_{n}(R) \backslash M_{n}(I)$ the corresponding matrix in $M_{n}(R / I)$ is $\left[a_{i j}+I\right]$. If $A=\left[a_{i j}\right] \in M_{n}(I)$, then the corresponding matrix in $M_{n}(R / I)$ is the zero matrix in $M_{n}(R / I)$. For convenience, we denote the matrix $\left[a_{i j}+I\right] \in M_{n}(R / I)$ as $\bar{A}$ corresponding to the matrix $A=\left(a_{i j}\right)$. In Section 2, we prove that for $n \geq 2, \Gamma_{I^{t}}\left(M_{n}(R)\right)$ is a connected graph of diameter 2 and of girth 3. In Section 3, we study the structure of $\Gamma_{I^{t}}\left(M_{n}(R)\right)$ through the relationship between $\Gamma_{I^{t}}\left(M_{n}(R)\right)$ and $\Gamma_{t}\left(M_{n}(R / I)\right)$. In Section 4, we discuss the clique, chromatic, and independence numbers of $\Gamma_{I^{t}}\left(M_{n}(R)\right)$.

\section{Girth and diameter}

In this section, we list some properties of the trace graph of matrix ring with respect to an ideal $I$ of $R$ that can be proved by similar arguments as in the case of the trace graph of matrix rings over commutative rings. For $A=\left[a_{i j}\right] \in M_{n}(R)$, we set $J_{I}(A)=\sum_{1 \leq i, j \leq n}(R / I)\left(a_{i j}+I\right) \in(R / I)$; the sum of the ideals of $R / I$ generated by all entries of $A=\left[a_{i j}+I\right]$ over $R / I$. Note that $J_{I}(A)$ is an ideal of $R / I$. 
Proposition 2.1. For a non-zero ideal $I$ of $R$ and an integer $\left.n \geq 2, \Gamma_{I^{t}}\left(M_{n}(R)\right)\right)$ contains no isolated vertex.

Proof. Let $A=\left[a_{i j}\right] \in M_{n}(R) \backslash M_{n}(I)$.

Case 1. If $A \neq I_{n}$ and $\operatorname{Tr}(A) \in I$, then $A$ is adjacent to the identity matrix $I_{n}$.

Case 2. Assume that $\operatorname{Tr}(A) \notin I$.

Case 2.1. Suppose $A$ has exactly one entry $a_{k \ell}$ such that $a_{k \ell} \notin I$. Choose $B=\left[b_{i j}\right]$ such that $b_{\ell k} \in I$ and $b_{i j} \notin I$ otherwise. Then $B \in M_{n}(R) \backslash M_{n}(I)$ and $\operatorname{Tr}(A B)=$ $a_{11} b_{11}+\cdots+a_{1 n} b_{n 1}+a_{21} b_{12}+\cdots+a_{2 n} b_{n 2}+\cdots+a_{n 1} b_{1 n}+\cdots+a_{n n} b_{n n}$. Note that in each term of $\operatorname{Tr}(A B)$ either $a_{i j} \in I$ or $b_{i j} \in I$. Since $I$ is an ideal of $R, a_{i j} b_{i j} \in I$ for every $1 \leq i, j \leq n$ and hence their sum belongs to $I$. Thus $\operatorname{Tr}(A B) \in I$.

Case 2.2. Suppose that $A$ has at least two entries $a_{k \ell}, a_{k_{1} \ell_{1}}$ which are not elements of $I$. Then choose $B=\left[b_{i j}\right]$ such that $b_{\ell k}=-a_{k_{1} \ell_{1}}, b_{\ell_{1} k_{1}}=a_{k \ell}, b_{i j} \in I$ elsewhere. Thus $B \in M_{n}(R) \backslash M_{n}(I)$ and $\operatorname{Tr}(A B)=a_{k \ell} b_{\ell k}+a_{k_{1} \ell_{1}} b_{\ell_{1} k_{1}}+$ elements of $I$. Hence $\operatorname{Tr}(A B) \in I$.

Thus in all the cases for every $A \in M_{n}(R) \backslash M_{n}(I)$, there exists $B \in M_{n}(R) \backslash M_{n}(I)$ such that $\operatorname{Tr}(A B) \in I$. Hence, $\left.\Gamma_{I^{t}}\left(M_{n}(R)\right)\right)$ contains no isolated vertex.

In the following, we prove that no vertex in $\left.\Gamma_{I^{t}}\left(M_{n}(R)\right)\right)$ is adjacent to all other vertices.

Proposition 2.2. For a non-zero ideal $I$ of $R$ and an integer $n \geq 2$, no vertex of $\Gamma_{I^{t}}\left(M_{n}(R)\right)$ is adjacent to every other vertex of $\Gamma_{I^{t}}\left(M_{n}(R)\right)$.

Proof. Given a matrix $A=\left[a_{i j}\right] \in M_{n}(R) \backslash M_{n}(I)$. There exists at least one entry $a_{k \ell}$ such that $a_{k \ell} \notin I$. Choose $B=\left[b_{i j}\right]$ such that $b_{\ell k}=1$ and $b_{i j}=0$ elsewhere. Thus $B \in V\left(\Gamma_{I^{t}}\left(M_{n}(R)\right)\right)$ and $\operatorname{Tr}(A B)=a_{k \ell} \notin I$. If $A=B$, then $\operatorname{Tr}\left(A I_{n}\right) \notin I$.

Now we obtain, the degree of vertices in $\Gamma_{I^{t}}\left(M_{n}(R)\right)$.

Proposition 2.3. Let $R$ be a finite commutative ring and $n \geq 2$ be an integer.

1. For any vertex $A$ of $\Gamma_{I^{t}}\left(M_{n}(R)\right)$, we have:

a. $\operatorname{deg}(A)=\frac{|R|^{n^{2}}}{\left|J_{I}(A)\right|}-1$ if $\operatorname{Tr}\left(A^{2}\right) \notin I$, and

b. $\operatorname{deg}(A)=\frac{|R|^{n^{2}}}{\left|J_{I}(A)\right|}-2$ if $\operatorname{Tr}\left(A^{2}\right) \in I$.

2. $\delta\left(\Gamma_{t}\left(M_{n}(R)\right)\right)=|R|^{n^{2}-1}|I|-2$.

Proof. 1. Let $A \in M_{n}(R)$. Consider $f_{A}: M_{n}(R) \rightarrow R$ defined by $f_{A}(B)=\operatorname{Tr}(A B)$ and natural homomorphism $\varphi: R \rightarrow R / I$ by $\varphi(x)=x+I$. Clearly $\varphi \circ f_{A}: M_{n}(R) \rightarrow R / I$ is a surjective homomorphism with $\left(\varphi \circ f_{A}\right)(B)=\operatorname{Tr}(A B)+I, \operatorname{Im}\left(\varphi \circ f_{A}\right)=J_{I}(A)$ and $\operatorname{ker}\left(\varphi \circ f_{A}\right)=\left\{B \in M_{n}(R) \mid \operatorname{Tr}(A B) \in I\right\}$.

By the isomorphism theorem, $\frac{M_{n}(R)}{\operatorname{ker}\left(\varphi \circ f_{A}\right)} \cong J_{I}(A)$ and so $\left|\operatorname{ker}\left(\varphi \circ f_{A}\right)\right|=\frac{\left|M_{n}(R)\right|}{\left|J_{I}(A)\right|}=\frac{\mid R n^{n^{2}}}{\left|J_{I}(A)\right|}$. When $\operatorname{Tr}\left(A^{2}\right) \notin I, \operatorname{ker}\left(\varphi \circ f_{A}\right)$ contains exactly the vertices adjacent to $A$ and the zero matrix. When $\operatorname{Tr}\left(A^{2}\right) \in I, \operatorname{ker}\left(\varphi \circ f_{A}\right)$ contains additionally $A$. Hence (a) and (b) hold.

2. Consider the matrix $A=\left[a_{i j}\right] \in M_{n}(R) \backslash M_{n}(I)$ with $a_{i i} \in I$ for every $1 \leq i \leq n, a_{i j} \notin I$ implies $a_{j i} \in I$ for every $i \neq j$ and $a_{i j}$ is a unit for some $i$ and $j$. Clearly $J_{I}(A)=R / I$ and $\operatorname{Tr}\left(A^{2}\right) \in I$. Thus by $1(\mathrm{~b})$, we have $\operatorname{deg}(A)=|R|^{n^{2}-1}|I|-2$ and so $\delta \leq|R|^{n^{2}-1}|I|-2$.

Since $\left|J_{I}(A)\right| \leq|R / I|$ for every ideal $J_{I}(A)$ of $R / I, \frac{|R|^{n^{2}}}{\left|J_{I}(A)\right|} \geq|R|^{n^{2}-1}|I|$. From this $|R|^{n^{2}-1}|I|-2 \leq \frac{|R|^{n^{2}}}{\left|J_{I}(A)\right|}-2 \leq \operatorname{deg}(A)$ for every $A \in M_{n}(R)$. Thus, $\delta=|R|^{n^{2}-1}|I|-2$. 
From Proposition 2.3, for a finite commutative ring $R, \Gamma_{I^{t}}\left(M_{n}(R)\right)$ can never be an Eulerian graph. For, consider the matrices $E_{11}$ and $E_{1 n}$ where $n \neq 1 . \operatorname{Tr}\left(E_{11}^{2}\right)=1 \notin I$ and $\operatorname{Tr}\left(E_{1 n}^{2}\right)=0 \in I$. Hence, by the Proposition 2.3, either of $E_{11}$ and $E_{1 n}$ must have odd degree.

Proposition 2.4. Let $R$ be a commutative ring, $n \geq 2$ be an integer and $I$ be a non trivial ideal of $R$. Then $\Gamma_{I^{t}}\left(M_{n}(R)\right)$ is connected with diam $\left(\Gamma_{I^{t}}\left(M_{n}(R)\right)\right)=2$ and $g r\left(\Gamma_{I^{t}}\left(M_{n}(R)\right)\right)$ $=3$.

Proof. Let $A=\left[a_{i j}\right]$ and $B=\left[b_{i j}\right]$ be two distinct elements of $M_{n}(R) \backslash M_{n}(I)$. If $\operatorname{Tr}(A B) \in$ $I$, then $d(A, B)=1$. Assume that $\operatorname{Tr}(A B) \notin I$. By Proposition 2.2, $\operatorname{diam}\left(\Gamma_{I^{t}}\left(M_{n}(R)\right)\right)>1$. Now let us consider two cases:

Case 1. Suppose $a_{i j} b_{k \ell}-a_{k \ell} b_{i j} \in I$ for each $(i, j),(k, l) \in\{1, \ldots, n\}^{2}$.

Let $\left(i_{0}, j_{0}\right)$ and $\left(i_{1}, j_{1}\right)$ be two distinct elements of $\{1, \ldots, n\}^{2}$ such that $a_{i_{0} j_{0}} \notin I$. Consider the matrix $C=\left[c_{i j}\right]$ with $c_{j_{0} i_{0}}=-a_{i_{1} j_{1}}, c_{j_{1} i_{1}}=a_{i_{0} j_{0}}$, and $c_{k \ell} \in I$ elsewhere. Then $C \in M_{n}(R) \backslash M_{n}(I)$ and

$$
\begin{aligned}
\operatorname{Tr}(A C) & =a_{i_{0} j_{0}} c_{j_{0} i_{0}}+a_{i_{1} j_{1}} c_{j_{1} i_{1}}+\text { elements of } I \\
& =-a_{i_{0} j_{0}} a_{i_{1} j_{1}}+a_{i_{1} j_{1}} a_{i_{0} j_{0}}+\text { elements of } I \in I
\end{aligned}
$$

and

$$
\begin{aligned}
\operatorname{Tr}(B C) & =b_{i_{0} j_{0}} c_{j_{0} i_{0}}+b_{i_{1} j_{1}} c_{j_{1} i_{1}}+\text { elements of } I \\
& =-b_{i_{0} j_{0}} a_{i_{1} j_{1}}+b_{i_{1} j_{1}} a_{i_{0} j_{0}}+\text { elements of } I \in I .
\end{aligned}
$$

Case 2. Suppose there exist $\left(i_{0}, j_{0}\right),\left(i_{1}, j_{1}\right) \in\{1, \ldots, n\}^{2}$ such that $a_{i_{0} j_{0}} b_{i_{1} j_{1}}-a_{i_{1} j_{1}} b_{i_{0} j_{0}} \notin$ $I$.

Let $\left(i_{2}, j_{2}\right) \in\{1, \ldots, n\}^{2} \backslash\left\{\left(i_{0}, j_{0}\right),\left(i_{1}, j_{1}\right)\right\}$ and consider the matrix $C=\left[c_{i j}\right]$ where

$$
\begin{aligned}
c_{j_{0} i_{0}} & =a_{i_{1} j_{1}} b_{i_{2} j_{2}}-a_{i_{2} j_{2}} b_{i_{1} j_{1}}, \\
c_{j_{1} i_{1}} & =a_{i_{2} j_{2}} b_{i_{0} j_{0}}-a_{i_{0} j_{0}} b_{i_{2} j_{2}}, \\
c_{j_{2} i_{2}} & =a_{i_{0} j_{0}} b_{i_{1} j_{1}}-a_{i_{1} j_{1}} b_{i_{0} j_{0}} \text { and } \\
c_{k \ell} & \in I \text { elsewhere. }
\end{aligned}
$$

Then $C \in M_{n}(R) \backslash M_{n}(I)$ and

$$
\begin{aligned}
\operatorname{Tr}(A C)= & a_{i_{0} j_{0}} c_{j_{0} i_{0}}+a_{i_{1} j_{1}} c_{j_{1} i_{1}}+a_{i_{2} j_{2}} c_{j_{2} i_{2}} \\
= & a_{i_{0} j_{0}} a_{i_{1} j_{1}} b_{i_{2} j_{2}}-a_{i_{0} j_{0}} a_{i_{2} j_{2}} b_{i_{1} j_{1}}+a_{i_{1} j_{1}} a_{i_{2} j_{2}} b_{i_{0} j_{0}} \\
& -a_{i_{1} j_{1}} a_{i_{0} j_{0}} b_{i_{2} j_{2}}+a_{i_{2} j_{2}} a_{i_{0} j_{0}} b_{i_{1} j_{1}}-a_{i_{2} j_{2}} a_{i_{1} j_{1}} b_{i_{0} j_{0}}+\text { elements of } I \in I,
\end{aligned}
$$

and $\operatorname{Tr}(B C)=b_{i_{0} j_{0}} c_{j 0} i_{0}+b_{i_{1} j_{1}} c_{j_{1} i_{1}}+b_{i_{2} j_{2}} c_{j_{2} i_{2}}$

$$
\begin{aligned}
= & b_{i_{0} j_{0}} a_{i_{1} j_{1}} b_{i_{2} j_{2}}-b_{i_{0} j_{0}} a_{i_{2} j_{2}} b_{i_{1} j_{1}}+b_{i_{1} j_{1}} a_{i_{2} j_{2}} b_{i_{0} j_{0}} \\
& -b_{i_{1} j_{1}} a_{i_{0} j_{0}} b_{i_{2} j_{2}}+b_{i_{2} j_{2}} a_{i_{0} j_{0}} b_{i_{1} j_{1}}-b_{i_{2} j_{2}} a_{i_{1} j_{1}} b_{i_{0} j_{0}}+\text { elements of } I \in I .
\end{aligned}
$$

In both cases, $A \neq C$ and $B \neq C$ (otherwise $\operatorname{Tr}(A B) \in I$ ) and hence $d(A, B)=2$. Consequently, $\Gamma_{I^{t}}\left(M_{n}(R)\right)$ is connected and $\operatorname{diam}\left(\Gamma_{I^{t}}\left(M_{n}(R)\right)\right)=2$.

Consider nonzero distinct matrices $A=\left[a_{i j}\right]$ with $a_{11}=1$ and $a_{i j} \in I$ elsewhere, $B=\left[b_{i j}\right]$ with $b_{n n}=1$ and $b_{i j} \in I$ elsewhere and $C=\left[c_{i j}\right]$ with $c_{1 n}=1$ and $c_{i j} \in I$ elsewhere. By the choice of $A, B, C$, we have $\operatorname{Tr}(A B), \operatorname{Tr}(B C), \operatorname{Tr}(A C) \in I$. Thus $A-B-C-A$ is a cycle, and so $\operatorname{gr}\left(\Gamma_{I^{t}}\left(M_{n}(R)\right)\right)=3$.

Remark 2.5. (i). By Propositions 2.2 and 2.4, the eccentricity of every vertex in $\Gamma_{I^{t}}\left(M_{n}(R)\right)$ is 2 and hence the radius of $\Gamma_{I^{t}}\left(M_{n}(R)\right)$ is 2 . i.e., the graph $\Gamma_{I^{t}}\left(M_{n}(R)\right)$ is self-centered.

(ii). By Proposition 2.4 $\Gamma_{I^{t}}\left(M_{n}(R)\right)$ contains an odd cycle, and so $\Gamma_{I^{t}}\left(M_{n}(R)\right)$ can never be a bipartite graph. 


\section{Relationship between $\Gamma_{I^{t}}\left(M_{n}(R)\right)$ and $\Gamma_{t}\left(M_{n}(R / I)\right)$}

In this section, we study the graph $\Gamma_{I^{t}}\left(M_{n}(R)\right)$ through $\Gamma_{t}\left(M_{n}(R / I)\right)$. The following theorem is useful in the further discussion of this paper.

Theorem 3.1. Let $R$ be a ring and $I$ be an ideal of $R$. Then $M_{n}(R) / M_{n}(I) \cong M_{n}(R / I)$.

Proof. The map $\varphi: M_{n}(R) / M_{n}(I) \rightarrow M_{n}(R / I)$ by $\left[a_{i j}\right]+M_{n}(I)=\left[a_{i j}+I\right]$ defines an isomorphism between $M_{n}(R) / M_{n}(I)$ and $M_{n}(R / I)$.

Note 3.2. From the isomorphism defined in Theorem 3.1, given an ideal $I$ of $R$ and a matrix $A \in M_{n}(R)$, we can view the trace of the coset $A+M_{n}(I)$ in $M_{n}(R) / M_{n}(I)$ as the trace of $\bar{A}$ in $M_{n}(R / I)$. Thus, the trace graph of $M_{n}(R) / M_{n}(I)$ is the trace graph of $M_{n}(R / I)$.

Theorem 3.3. Let $I$ be an ideal of a commutative ring $R, n \geq 2$ be a positive integer and $A=\left[a_{i j}\right], B=\left[b_{i j}\right] \in M_{n}(R) \backslash M_{n}(I)$ Then the following are true:

1. If $\bar{A}$ is adjacent to $\bar{B}$ in $\Gamma_{t}\left(M_{n}(R / I)\right)$, then $A$ and $B$ are adjacent in $\Gamma_{I^{t}}\left(M_{n}(R)\right)$.

2. If $A$ is adjacent to $B$ in $\Gamma_{I^{t}}\left(M_{n}(R)\right)$ and $\bar{A} \neq \bar{B}$, then $\bar{A}$ is adjacent to $\bar{B}$ in $\Gamma_{t}\left(M_{n}(R / I)\right)$.

3. If $A$ is adjacent to $B$ in $\Gamma_{I^{t}}\left(M_{n}(R)\right)$ and $\bar{A}=\bar{B}$, then $\operatorname{Tr}\left(A^{2}\right), \operatorname{Tr}\left(B^{2}\right) \in I$.

4. If $\operatorname{Tr}\left(A^{2}\right) \in I$ and $\bar{A}=\bar{B}$, then $A$ is adjacent to $B$ in $\Gamma_{I^{t}}\left(M_{n}(R)\right)$ and $\operatorname{Tr}\left(B^{2}\right) \in I$.

5. If $A$ and $B$ are (distinct) adjacent vertices in $\Gamma_{I^{t}}\left(M_{n}(R)\right)$, then all (distinct) elements of $\bar{A}$ are adjacent to all elements of $\bar{B}$ in $\Gamma_{I^{t}}\left(M_{n}(R)\right)$. In particular, if $\operatorname{Tr}\left(A^{2}\right) \in I$, then all the distinct elements of $\bar{A}$ are adjacent in $\Gamma_{I^{t}}\left(M_{n}(R)\right)$.

Proof. 1. In view of the fact mentioned in Note 3.2 , it is enough to prove that $A+M_{n}(I)$ is adjacent to $B+M_{n}(I)$ in $\Gamma_{t}\left(M_{n}(R) / M_{n}(I)\right)$ implies $A$ is adjacent to $B$ in $\Gamma_{I^{t}}\left(M_{n}(R)\right)$. When $A+M_{n}(I)$ is adjacent to $B+M_{n}(I)$ in $\Gamma_{t}\left(M_{n}(R) / M_{n}(I)\right)$, we have $\operatorname{Tr}(A B+$ $\left.M_{n}(I)\right)=M_{n}(I)$ and so $\operatorname{Tr}(A B) \in I$. Thus $A$ is adjacent to $B$ in $\Gamma_{I^{t}}\left(M_{n}(R)\right)$.

2. If $A$ is adjacent to $B$ in $\Gamma_{I^{t}}\left(M_{n}(R)\right)$, then $\operatorname{Tr}(A B) \in I$. This gives that $\operatorname{Tr}(A B)+I=I$ and hence $\operatorname{Tr}\left(A B+M_{n}(I)\right)=M_{n}(I)$. Thus, $\operatorname{Tr}\left(\left(A+M_{n}(I)\right)\left(B+M_{n}(I)\right)\right)=M_{n}(I)$, and so $A+M_{n}(I)$ is adjacent to $B+M_{n}(I)$ in $\Gamma_{t}\left(M_{n}(R) / M_{n}(I)\right)$.

3. If $A$ is adjacent to $B$ in $\Gamma_{I^{t}}\left(M_{n}(R)\right)$, by (2) above $\operatorname{Tr}\left(\left(A+M_{n}(I)\right)\left(B+M_{n}(I)\right)\right)=$ $M_{n}(I)$. Since $A+M_{n}(I)=B+M_{n}(I), \operatorname{Tr}\left(\left(A+M_{n}(I)\right)\left(A+M_{n}(I)\right)\right)=M_{n}(I)$. i.e., $\operatorname{Tr}\left(A^{2}+M_{n}(I)\right)=M_{n}(I)$ giving $\operatorname{Tr}\left(A^{2}\right)+I=I$. Thus $\operatorname{Tr}\left(A^{2}\right) \in I$ and similarly $\operatorname{Tr}\left(B^{2}\right) \in I$.

4. If $\operatorname{Tr}\left(A^{2}\right) \in I$, then $\operatorname{Tr}\left(A^{2}\right)+I=I$ and so $\operatorname{Tr}\left(A^{2}+M_{n}(I)\right)=M_{n}(I)$. $\operatorname{Thus} \operatorname{Tr}((A+$ $\left.\left.M_{n}(I)\right)\left(B+M_{n}(I)\right)\right)=M_{n}(I)$ giving $\operatorname{Tr}(A B)+I=I$. Thus $\operatorname{Tr}(A B) \in I$. i.e., $A$ is adjacent to $B$ in $\Gamma_{I^{t}}\left(M_{n}(R)\right)$. By $(3), \operatorname{Tr}\left(B^{2}\right) \in I$.

5. It is enough to prove that if $A$ and $B$ are (distinct) adjacent vertices in $\Gamma_{I^{t}}\left(M_{n}(R)\right)$, then all (distinct) elements of $A+M_{n}(I)$ are adjacent to all elements of $B+M_{n}(I)$ in $\Gamma_{I^{t}}\left(M_{n}(R)\right)$. In particular, if $\operatorname{Tr}\left(A^{2}\right) \in I$, then all the distinct elements of $A+M_{n}(I)$ are adjacent in $\Gamma_{I^{t}}\left(M_{n}(R)\right)$.

By (1) and (2), if $A$ and $B$ are adjacent vertices in $\Gamma_{I^{t}}\left(M_{n}(R)\right)$, then all (distinct) elements of $A+M_{n}(I)$ and $B+M_{n}(I)$ are adjacent in $\Gamma_{I^{t}}\left(M_{n}(R)\right)$. As a particular case, taking $B=A$, we get if $\operatorname{Tr}\left(A^{2}\right) \in I$, then all the distinct elements of $A+M_{n}(I)$ are adjacent in $\Gamma_{I^{t}}\left(M_{n}(R)\right)$.

Corollary 3.4. Let $I$ be an ideal of a commutative ring $R$ and $n \geq 2$ be a positive integer. Then $\Gamma_{I^{t}}\left(M_{n}(R)\right)$ contains $\left|M_{n}(I)\right|$ disjoint subgraphs each isomorphic to $\Gamma_{t}\left(M_{n}(R / I)\right)$.

Proof. Let $\left\{A_{i}\right\}_{i \in \Lambda}$ be distinct coset representatives of elements in the quotient ring $M_{n}(R) / M_{n}(I)$. Then the vertex set of $\Gamma_{t}\left(M_{n}(R) / M_{n}(I)\right)$ is partitioned into $\left\{A_{i}+M_{n}(I)\right\}_{i \in \Lambda}$. 
Note that $A_{i}+M_{n}(I) \neq A_{j}+M_{n}(I)$ for $i \neq j$. Fix $X \in M_{n}(I)$. Consider the subgraph $H_{X}$ with vertex set $\left\{A_{i}+X: i \in \Lambda\right\} \subseteq V\left(\Gamma_{I^{t}}\left(M_{n}(R)\right)\right)$ and two vertices $A_{i}+X$ and $A_{j}+X$ are adjacent in $H_{X}$ if $A_{i}+M_{n}(I)$ and $A_{j}+M_{n}(I)$ are adjacent in $\Gamma_{t}\left(M_{n}(R) / M_{n}(I)\right)$. Clearly, $H_{X}$ is isomorphic to $\Gamma_{t}\left(M_{n}(R) / M_{n}(I)\right)$.

Assume that $A_{i}+X$ and $A_{j}+X$ are adjacent in $H_{X}$. By the definition of $H_{X}, A_{i}+$ $M_{n}(I)$ is adjacent to $A_{j}+M_{n}(I)$ in $\Gamma_{t}\left(M_{n}(R) / M_{n}(I)\right)$. By Theorem 3.3(1), $A_{i}$ and $A_{j}$ are adjacent in $\Gamma_{I^{t}}\left(M_{n}(R)\right)$. By Theorem 3.3(4), $A_{i}+X$ and $A_{j}+X$ are adjacent in $\Gamma_{I^{t}}\left(M_{n}(R)\right)$. Hence $H_{X}$ is a subgraph of $\Gamma_{I^{t}}\left(M_{n}(R)\right)$.

Also, for any $Y(\neq X) \in M_{n}(I), V\left(H_{X}\right) \cap V\left(H_{Y}\right)=\phi$. Thus, $\Gamma_{I^{t}}\left(M_{n}(R)\right)$ contains $\left|M_{n}(I)\right|$ disjoint subgraphs each isomorphic to $\Gamma_{t}\left(M_{n}(R) / M_{n}(I)\right)$ and so contains $\left|M_{n}(I)\right|$ disjoint subgraphs isomorphic to $\Gamma_{t}\left(M_{n}(R / I)\right)$.

Remark 3.5. The following are true:

1. $\Gamma_{t}\left(M_{n}(R) / M_{n}(I)\right)$ is a graph with $\left|M_{n}(R / I)\right|-1$ vertices.

2. $\Gamma_{I^{t}}\left(M_{n}(R)\right)$ is a graph with $\left|M_{n}(R)\right|-\left|M_{n}(I)\right|$ vertices.

3. Let $R$ be a finite commutative ring. Note that Corollary 3.4 exhibits a partition of $\Gamma_{I^{t}}\left(M_{n}(R)\right)$ into vertex disjoint subgraphs. Thus

$$
\left|M_{n}(I)\right|\left|V\left(\Gamma_{t}\left(M_{n}(R / I)\right)\right)\right|=\left|V\left(\Gamma_{I^{t}}\left(M_{n}(R)\right)\right)\right| .
$$

The following theorem puts forth a partition of $\Gamma_{I^{t}}\left(M_{n}(R)\right)$ into edge disjoint subgraphs. In view of Proposition 3.3(4), if $\operatorname{Tr}\left(A^{2}\right) \in I$ and $\bar{A}=\bar{B}$, then $A$ is adjacent to $B$ in $\Gamma_{I^{t}}\left(M_{n}(R)\right)$ and $\operatorname{Tr}\left(B^{2}\right) \in I$. This means that if $\operatorname{Tr}\left(A^{2}\right) \in I$ for a matrix $A$, then the same is true for all matrices in the coset of $A$.

Theorem 3.6. Let $R$ be a commutative ring with identity, $I$ be a non trivial ideal of $R$, $n \geq 2$ be an integer and

$$
\lambda=\mid\left\{\bar{A} \in V\left(\Gamma_{t}\left(M_{n}(R / I)\right)\right): \operatorname{Tr}\left(A^{2}\right) \in I \text { and } A \text { is a coset representative of } \bar{A}\right\} \mid .
$$

Then $\Gamma_{I^{t}}\left(M_{n}(R)\right) \cong\left|M_{n}(I)\right|^{2} \Gamma_{t}\left(M_{n}(R / I)\right) \oplus \lambda K_{\left|M_{n}(I)\right|}$.

Proof. Consider the partition of edges of $\Gamma_{t}\left(M_{n}(R / I)\right)$ given below:

$$
\begin{aligned}
& E_{1}=\left\{e=(\bar{A}, \bar{B}): \operatorname{Tr}\left(A^{2}\right), \operatorname{Tr}\left(B^{2}\right) \notin I\right\} \\
& E_{2}=\left\{e=(\bar{A}, \bar{B}): \operatorname{Tr}\left(A^{2}\right), \operatorname{Tr}\left(B^{2}\right) \in I\right\} \\
& E_{3}=\left\{e=(\bar{A}, \bar{B}): \text { either } \operatorname{Tr}\left(A^{2}\right) \text { or } \operatorname{Tr}\left(B^{2}\right) \in I\right\} .
\end{aligned}
$$

Let $e=(\bar{A}, \bar{B}) \in E\left(\Gamma_{t}\left(M_{n}(R / I)\right)\right)$. By Theorem 3.3(1) and (4), the subgraph induced by the set $V_{e}=\left\{A+N_{1}, B+N_{2}: N_{1}, N_{2} \in M_{n}(I)\right\}$ in $\Gamma_{I^{t}}\left(M_{n}(R)\right)$ is

$$
\left\langle V_{e}\right\rangle= \begin{cases}K_{\left|M_{n}(I)\right|,\left|M_{n}(I)\right|} & \text { if } \operatorname{Tr}\left(A^{2}\right), \operatorname{Tr}\left(B^{2}\right) \notin I ; \\ K_{\left|M_{n}(I)\right|,\left|M_{n}(I)\right|} \oplus 2 K_{\left|M_{n}(I)\right|} & \text { if } \operatorname{Tr}\left(A^{2}\right), \operatorname{Tr}\left(B^{2}\right) \in I ; \\ K_{\left|M_{n}(I)\right|,\left|M_{n}(I)\right|} \oplus K_{\left|M_{n}(I)\right|} & \text { if either } \operatorname{Tr}\left(A^{2}\right) \text { or } \operatorname{Tr}\left(B^{2}\right) \in I .\end{cases}
$$

By [4, p.192], we have $K_{\left|M_{n}(I)\right|,\left|M_{n}(I)\right|} \cong M_{1}^{(e)} \oplus \cdots \oplus M_{\left|M_{n}(I)\right|}^{(e)}$, where each of $M_{i}^{(e)}$ is a perfect matching of $K_{\left|M_{n}(I)\right|,\left|M_{n}(I)\right|}$. Thus,

$$
\left\langle V_{e}\right\rangle= \begin{cases}M_{1}^{(e)} \oplus \cdots \oplus M_{\left|M_{n}(I)\right|}^{(e)} & \text { if } \operatorname{Tr}\left(A^{2}\right), \operatorname{Tr}\left(B^{2}\right) \notin I \\ M_{1}^{(e)} \oplus \cdots \oplus M_{\left|M_{n}(I)\right|}^{(e)} \oplus 2 K_{\left|M_{n}(I)\right|} & \text { if } \operatorname{Tr}\left(A^{2}\right), \operatorname{Tr}\left(B^{2}\right) \in I \\ M_{1}^{(e)} \oplus \cdots \oplus M_{\left|M_{n}(I)\right|}^{(e)} \oplus K_{\left|M_{n}(I)\right|} & \text { if either } \operatorname{Tr}\left(A^{2}\right) \text { or } \operatorname{Tr}\left(B^{2}\right) \in I .\end{cases}
$$

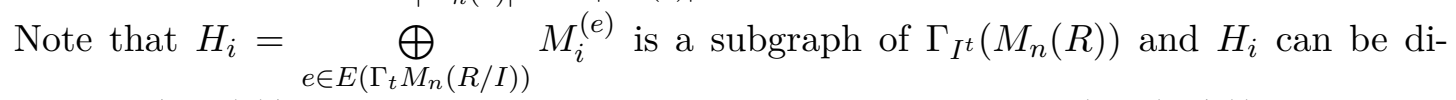
vided into $\left|M_{n}(I)\right|$ edge disjoint subgraphs each isomorphic to $\Gamma_{t}\left(M_{n}(R / I)\right)$, i.e., $H_{i} \cong$ $\left|M_{n}(I)\right| \Gamma_{t}\left(M_{n}(R / I)\right)$.

Clearly $H=H_{1} \oplus \cdots \oplus H_{\left|M_{n}(I)\right|}$ is a subgraph with vertex set $M_{n}(R) \backslash M_{n}(I)$ and $H \cong\left|M_{n}(I)\right|^{2} \Gamma_{t}\left(M_{n}(R / I)\right)$. Thus $\Gamma_{I^{t}}\left(M_{n}(R)\right) \cong\left|M_{n}(I)\right|^{2} \Gamma_{t}\left(M_{n}(R / I)\right) \oplus \lambda K_{\left|M_{n}(I)\right|}$ where $\lambda=\mid\left\{\bar{A} \in V\left(\Gamma_{t}\left(M_{n}(R / I)\right): \operatorname{Tr}\left(A^{2}\right) \in I\right.\right.$ and $A$ is a coset representative of $\left.\bar{A}\right\} \mid$. 


\section{Chromatic, clique and independence numbers of $\Gamma_{I^{t}}\left(M_{n}(R)\right)$}

In this section, we obtain bounds for the clique, chromatic and independence numbers of $\Gamma_{I^{t}}\left(M_{n}(R)\right)$ and obtain a condition for the chromatic and clique numbers of $\Gamma_{I^{t}}\left(M_{n}(R)\right)$ to be equal.

Theorem 4.1. Let $n \geq 2$ be an integer, $R$ be a commutative ring and $I$ be a non trivial ideal of $R$. Then the following hold:

1. $\omega\left(\Gamma_{t}\left(M_{n}(R / I)\right)\right) \leq \omega\left(\Gamma_{I^{t}}\left(M_{n}(R)\right)\right) \leq\left|M_{n}(I)\right| \omega\left(\Gamma_{t}\left(M_{n}(R / I)\right)\right)$. Moreover, the equality $\omega\left(\Gamma_{I^{t}}\left(M_{n}(R)\right)\right)=\left|M_{n}(I)\right| \omega\left(\Gamma_{t}\left(M_{n}(R / I)\right)\right)$ holds if there exists a clique of maximum order in $\Gamma_{t}\left(M_{n}(R / I)\right)$ such that $\operatorname{Tr}\left(A^{2}\right) \in I$ for every vertex $\bar{A}$ in the clique.

2. $\chi\left(\Gamma_{t}\left(M_{n}(R / I)\right)\right) \leq \chi\left(\Gamma_{I^{t}}\left(M_{n}(R)\right)\right) \leq\left|M_{n}(I)\right| \chi\left(\Gamma_{t}\left(M_{n}(R / I)\right)\right)$.

Proof. 1. The first inequality follows from the fact that $\Gamma_{t}\left(M_{n}(R / I)\right)$ is a subgraph of $\Gamma_{I^{t}}\left(M_{n}(R)\right)$. Let $\omega\left(\Gamma_{t}\left(M_{n}(R / I)\right)\right)=k$. To conclude the proof, it is enough to prove that $\omega\left(\Gamma_{I^{t}}\left(M_{n}(R)\right)\right) \leq k\left|M_{n}(I)\right|$. Since $\Gamma_{t}\left(M_{n}(R / I)\right) \cong \Gamma_{t}\left(M_{n}(R) / M_{n}(I)\right)$, we have $\omega\left(\Gamma_{t}\left(M_{n}(R) / M_{n}(I)\right)\right)=k$.

Suppose there exists a clique of order $k\left|M_{n}(I)\right|+1$ in $\Gamma_{I^{t}}\left(M_{n}(R)\right)$. Let $\left\{B_{1}, \ldots\right.$, $\left.B_{k\left|M_{n}(I)\right|+1}\right\}$ be a clique in $\Gamma_{I^{t}}\left(M_{n}(R)\right)$. Consider the set

$$
X=\left\{B_{1}+M_{n}(I), \ldots, B_{k\left|M_{n}(I)\right|+1}+M_{n}(I)\right\} \subseteq V\left(\Gamma_{t}\left(M_{n}(R) / M_{n}(I)\right)\right) .
$$

Since $B_{i}$ is adjacent to $B_{j}$ in $\Gamma_{I^{t}}\left(M_{n}(R)\right)$, for $i \neq j$, either $B_{i}+M_{n}(I)=B_{j}+M_{n}(I)$ or $B_{i}+M_{n}(I)$ is adjacent to $B_{j}+M_{n}(I)$ in $\Gamma_{t}\left(M_{n}(R) / M_{n}(I)\right)$. Since $\left|B_{i}+M_{n}(I)\right|=\left|M_{n}(I)\right|$ we have at least $k+1$ distinct elements in $X$ such that the $k+1$ elements are adjacent to each other in $\Gamma_{t}\left(M_{n}(R) / M_{n}(I)\right)$. Thus $\omega\left(\Gamma_{t}\left(M_{n}(R) / M_{n}(I)\right)\right) \geq k+1$, which is a contradiction. Hence $\omega\left(\Gamma_{I^{t}}\left(M_{n}(R)\right)\right) \leq k\left|M_{n}(I)\right|$. The moreover case is clear from the preceding arguments and Theorem 3.3(1) and (5).

2. The first inequality is clear since $\Gamma_{t}\left(M_{n}(R / I)\right)$ is a subgraph of $\Gamma_{I^{t}}\left(M_{n}(R)\right)$. Let $\chi\left(\Gamma_{t}\left(M_{n}(R / I)\right)\right)=k$ and $C_{1}, \ldots, C_{k}$ be the color classes of $\Gamma_{t}\left(M_{n}(R / I)\right)$. Consider $\bar{A} \in$ $\Gamma_{t}\left(M_{n}(R / I)\right)$ belongs to the color class $C_{1}$ and the set $X_{A}=\left\{\left[a_{i j}\right] \in \Gamma_{I^{t}}\left(M_{n}(R)\right):\left[a_{i j}+\right.\right.$ $I]=\bar{A}\}$. Note that $\left|X_{A}\right|=\left|M_{n}(I)\right|$. Assign $\left|M_{n}(I)\right|$ distinct colors $C_{11}, \ldots, C_{1\left|M_{n}(I)\right|}$ to the vertices of $X_{A}$. Assign the same colors $C_{11}, \ldots, C_{1\left|M_{n}(I)\right|}$ for the vertices arising out of other vertices $\bar{B} \in \Gamma_{t}\left(M_{n}(R / I)\right)$ belonging to the color class $C_{1}$. Since $\bar{A}$ is not adjacent to $\bar{B}$ no vertex of $X_{A}$ is adjacent to $X_{B}$. Similarly for $2 \leq i \leq k$ assigning colors, $C_{i 1}, \ldots, C_{i\left|M_{n}(I)\right|}$ to the vertices of $\Gamma_{I^{t}}\left(M_{n}(R)\right)$ arising out of the vertices of the color class $C_{i}$ we have $k\left|M_{n}(I)\right|$ colors and the coloring is proper. Thus $\chi\left(\Gamma_{I^{t}}\left(M_{n}(R)\right)\right) \leq k\left|M_{n}(I)\right|$.

The following theorem is a generalization of the moreover case of Theorem 4.1(1).

Theorem 4.2. Let $n \geq 2$ be an integer, $R$ be a commutative ring and $I$ be a non trivial ideal in $R$. Let $S$ be a clique of maximum order in $\Gamma_{t}\left(M_{n}(R / I)\right)$ and $S$ have the largest number of elements $\bar{A}$ with $\operatorname{Tr}\left(A^{2}\right) \in I$. Let $X=\left\{\bar{A} \in S: \operatorname{Tr}\left(A^{2}\right) \notin I\right\}$. Then $\omega\left(\Gamma_{I^{t}}\left(M_{n}(R)\right)\right)=|X|+\left|M_{n}(I)\right|\left(\omega\left(\Gamma_{t}\left(M_{n}(R / I)\right)\right)-|X|\right)$.

Proof. Let $|X|=\left|\left\{\bar{A} \in S: \operatorname{Tr}\left(A^{2}\right) \notin I\right\}\right|=k_{1},\left|\left\{\bar{A} \in S: \operatorname{Tr}\left(A^{2}\right) \in I\right\}\right|=k_{2}$ and $\omega\left(\Gamma_{t}\left(M_{n}(R / I)\right)\right)=|S|=k$. Then $k_{1}+k_{2}=k$. In view of Note $3.2, \Gamma_{t}\left(M_{n}(R / I)\right) \cong$ $\Gamma_{t}\left(M_{n}(R) / M_{n}(I)\right)$ and so $\omega\left(\Gamma_{t}\left(M_{n}(R) / M_{n}(I)\right)\right)=k$.

Further by our assumption on $S$, any maximal clique of $\Gamma_{t}\left(M_{n}(R / I)\right)$ and hence of $\Gamma_{t}\left(M_{n}(R) / M_{n}(I)\right)$ can have at most $k_{2}$ number of vertices $\bar{A}$ with $\operatorname{Tr}\left(A^{2}\right) \in I$.

Hence one can take the clique corresponding to $S$ of $\Gamma_{t}\left(M_{n}(R / I)\right)$ as a clique $<\left\{A_{1}+\right.$ $\left.M_{n}(I), \ldots, A_{k}+M_{n}(I)\right\}>$ of $\Gamma_{t}\left(M_{n}(R) / M_{n}(I)\right)$ with $\operatorname{Tr}\left(A_{i}^{2}\right) \in I$ for $1 \leq i \leq k_{2}$ and $\operatorname{Tr}\left(A_{i}^{2}\right) \notin I$ for $k_{2}+1 \leq i \leq k$. Clearly the set $\left\{A_{i j}: A_{i j} \in A_{i}+M_{n}(I), 1 \leq i \leq\right.$ 
$k_{2}$ and $\left.1 \leq j \leq\left|M_{n}(I)\right|\right\} \cup\left\{A_{i 2}=A_{i}: k_{2}+1 \leq i \leq k\right\}$ is a clique of size $\left|M_{n}(I)\right| k_{2}+k_{1}$ in $\omega\left(\Gamma_{I^{t}}\left(M_{n}(R)\right)\right)$.

Hence $\omega\left(\Gamma_{I^{t}}\left(M_{n}(R)\right)\right) \geq k_{1}+\left|M_{n}(I)\right| k_{2}$.

To prove our result, it is enough to prove that $\omega\left(\Gamma_{I^{t}}\left(M_{n}(R)\right)\right) \leq k_{1}+\left|M_{n}(I)\right| k_{2}$. Suppose $\Gamma_{I^{t}}\left(M_{n}(R)\right)$ has a clique $S^{\prime}$ of order $k_{1}+\left|M_{n}(I)\right| k_{2}+1$. Without loss of generality, we may assume that $S^{\prime}$ is a maximal clique of order $\geq k_{1}+\left|M_{n}(I)\right| k_{2}+1$ in $\Gamma_{I^{t}}\left(M_{n}(R)\right)$. Let $A \in S^{\prime}$ with $\operatorname{Tr}\left(A^{2}\right) \notin I$.

By Theorem $3.3(3)$, no vertex in the set $\left\{A+B: B \in M_{n}(I)^{*}\right\}$ is adjacent to $A$. Hence $\left\{A+B: B \in M_{n}(I)^{*}\right\}$ has no intersection with $S^{\prime}$. Also note that due to the maximality of the clique $S^{\prime}$, if $A \in S^{\prime}$ with $\operatorname{Tr}\left(A^{2}\right) \in I$ then by Theorem 3.3 (2) and (4), the set $\left\{A+B: B \in M_{n}(I)\right\} \subset S^{\prime}$.

If $S^{\prime}$ contains at least $k_{2}\left|M_{n}(I)\right|+1$ vertices with $\operatorname{Tr}\left(A^{2}\right) \in I$, then by Theorem $3.3(2)$, the clique $S_{I}^{\prime}$ of $\Gamma_{t}\left(M_{n}(R) / M_{n}(I)\right)$ with respect to $S^{\prime}$ contains at least $k_{2}+1$ vertices with $\operatorname{Tr}\left(A^{2}\right) \in I$ which is a contradiction to our assumption that among the cliques of $\Gamma_{t}\left(M_{n}(R / I)\right), S$ has the largest number of elements $\bar{A}$ with $\operatorname{Tr}\left(A^{2}\right) \in I$.

Hence the number of vertices in $S^{\prime}$ with $\operatorname{Tr}\left(A^{2}\right) \in I$ is less than or equal to $k_{2}\left|M_{n}(I)\right|$. i.e., $S^{\prime}$ contains at least $k_{1}+1$ vertices with $\operatorname{Tr}\left(A^{2}\right) \notin I$. Now, the clique $S_{I}^{\prime}$ of $\Gamma_{t}\left(M_{n}(R) / M_{n}(I)\right)$ corresponding to $S^{\prime}$ contains at least $k_{2}+k_{1}+1$ vertices which is a contradiction to $\omega\left(\Gamma_{t}\left(M_{n}(R / I)\right)\right)=k$. Thus $\omega\left(\Gamma_{I^{t}}\left(M_{n}(R)\right)\right) \leq k_{1}+\left|M_{n}(I)\right| k_{2}$ and hence $\omega\left(\Gamma_{I^{t}}\left(M_{n}(R)\right)\right)=$ $k_{1}+\left|M_{n}(I)\right| k_{2}$.

Theorem 4.3. Let $n \geq 2$ be an integer, $R$ be a commutative ring and $I$ be a non trivial ideal of $R$. Let $\Gamma_{t}\left(M_{n}(R / I)\right)$ contain a clique of maximum order such that $\operatorname{Tr}\left(A^{2}\right) \in I$ for every $\bar{A}$ in the clique. If $\chi\left(\Gamma_{t}\left(M_{n}(R / I)\right)\right)=\omega\left(\Gamma_{t}\left(M_{n}(R / I)\right)\right)$, then $\chi\left(\Gamma_{I^{t}}\left(M_{n}(R)\right)\right)=$ $\omega\left(\Gamma_{I^{t}}\left(M_{n}(R)\right)\right)$.

Proof. Firstly, let us assume that $\chi\left(\Gamma_{t}\left(M_{n}(R / I)\right)\right)=\omega\left(\Gamma_{t}\left(M_{n}(R / I)\right)\right)=k$. From this we have $\chi\left(\Gamma_{t}\left(M_{n}(R) / M_{n}(I)\right)\right)=\omega\left(\Gamma_{t}\left(M_{n}(R) / M_{n}(I)\right)\right)=k$.

Let $\left\{A_{1}+M_{n}(I), \ldots, A_{k}+M_{n}(I)\right\}$ be a clique of order $k$ in $\Gamma_{t}\left(M_{n}(R) / M_{n}(I)\right)$ such that $\operatorname{Tr}\left(A_{i}^{2}\right) \in I, 1 \leq i \leq k$. Let $\left\{c_{1}, \ldots, c_{k}\right\}$ be a set of minimum colors required for a proper coloring of the graph $\Gamma_{t}\left(M_{n}(R) / M_{n}(I)\right)$. Without loss of generality assume that $A_{i}+M_{n}(I)$ is colored by the color $c_{i}$. Since $A_{i}^{2} \in I$, the set $X=\left\{A \in \Gamma_{I^{t}}\left(M_{n}(R)\right): A \in\right.$ $A_{i}+M_{n}(I)$ for some $\left.i \in\{1, \ldots, k\}\right\}$ forms a clique of order $\left|M_{n}(I)\right| k$ in $\Gamma_{I^{t}}\left(M_{n}(R)\right)$.

By Theorem 4.1(1), this clique is maximum and $\omega\left(\Gamma_{I^{t}}\left(M_{n}(R)\right)\right)=k\left|M_{n}(I)\right|$. Assign $k\left|M_{n}(I)\right|$ distinct colors $c_{1}^{\prime}, \ldots, c_{k\left|M_{n}(I)\right|}^{\prime}$ to the vertices in the set $X$.

For a vertex $B \in V\left(\Gamma_{I^{t}}\left(M_{n}(R)\right)\right) \backslash X$, there exists $M \in M_{n}(I)$ such that $B=B_{\ell}+M \in$ $B_{\ell}+M_{n}(I)$ for some $B_{\ell}+M_{n}(I) \notin\left\{A_{1}+M_{n}(I), \ldots, A_{k}+M_{n}(I)\right\}$. Let $c_{j}$ be the color of $B_{\ell}+M_{n}(I)$ in $\Gamma_{t}\left(M_{n}(R) / M_{n}(I)\right)$. Note that $A_{j}+M_{n}(I)$ belongs to the color class $c_{j}$ in $\Gamma_{t}\left(M_{n}(R) / M_{n}(I)\right)$.

Assign the color of $A_{j}+M$ in $\Gamma_{I^{t}}\left(M_{n}(R)\right)$ to $B=B_{\ell}+M$ in $\Gamma_{I^{t}}\left(M_{n}(R)\right)$. Let $C \in$ $V\left(\Gamma_{I^{t}}\left(M_{n}(R)\right)\right) \backslash X$ be adjacent to $B \in V\left(\Gamma_{I^{t}}\left(M_{n}(R)\right)\right) \backslash X$. Then $B_{\ell}+M_{n}(I)$ is adjacent to $C+M_{n}(I)$ in $\Gamma_{t}\left(M_{n}(R) / M_{n}(I)\right)$ and hence they belong to different color classes in $\Gamma_{t}\left(M_{n}(R) / M_{n}(I)\right)$ and so $B$ and $C$ belong to different color classes in $\Gamma_{I^{t}}\left(M_{n}(R)\right)$.

Thus we have given a proper coloring for the graph $\Gamma_{I^{t}}\left(M_{n}(R)\right)$ with $k\left|M_{n}(I)\right|$ colors and so $\chi\left(\Gamma_{I^{t}}\left(M_{n}(R)\right)\right) \leq k\left|M_{n}(I)\right|$. Since $k\left|M_{n}(I)\right|=\omega\left(\Gamma_{I^{t}}\left(M_{n}(R)\right)\right) \leq \chi\left(\Gamma_{I^{t}}\left(M_{n}(R)\right)\right)$, $\chi\left(\Gamma_{I^{t}}\left(M_{n}(R)\right)\right)=k\left|M_{n}(I)\right|$. 
Theorem 4.4. Let $n \geq 2$ be an integer, $R$ be a commutative ring and $I$ be a non trivial ideal of $R$. Then

1. $\alpha\left(\Gamma_{t}\left(M_{n}(R / I)\right)\right) \leq \alpha\left(\Gamma_{I^{t}}\left(M_{n}(R)\right)\right) \leq\left|M_{n}(I)\right| \alpha\left(\Gamma_{t}\left(M_{n}(R / I)\right)\right)$;

2. In particular, if there exists an independent set of maximum order in $\Gamma_{t}\left(M_{n}(R / I)\right)$ such that $\operatorname{Tr}\left(A^{2}\right) \notin I$ for every vertex $A$ in the independent set, then $\alpha\left(\Gamma_{I^{t}}\left(M_{n}(R)\right)\right)$ $=\left|M_{n}(I)\right| \alpha\left(\Gamma_{t}\left(M_{n}(R / I)\right)\right)$.

Proof. 1. Let $\alpha\left(\Gamma_{t}\left(M_{n}(R / I)\right)\right)=k$ and $X$ be the corresponding maximum independent set of $\Gamma_{t}\left(M_{n}(R / I)\right)$. Consider the set $X_{1}=\{A: \bar{A} \in X\} \subseteq V\left(\Gamma_{I^{t}}\left(M_{n}(R)\right)\right)$. By the Theorem 3.3(2), we have that $X_{1}$ is an independent set of order $k$ in $\Gamma_{I^{t}}\left(M_{n}(R)\right)$. Hence $\alpha\left(\Gamma_{t}\left(M_{n}(R / I)\right)\right) \leq \alpha\left(\Gamma_{I^{t}}\left(M_{n}(R)\right)\right)$. By Note 3.2 , we have $\alpha\left(\Gamma_{t}\left(M_{n}(R) / M_{n}(I)\right)\right)=k$.

Suppose that there exists an independent set of order $k\left|M_{n}(I)\right|+1$ in $\Gamma_{I^{t}}\left(M_{n}(R)\right)$. Let $\left\{B_{1}, \ldots, B_{k\left|M_{n}(I)\right|+1}\right\}$ be an independent set in $\Gamma_{I^{t}}\left(M_{n}(R)\right)$. Consider the set $X=$ $\left\{B_{1}+M_{n}(I), \ldots, B_{k\left|M_{n}(I)\right|+1}+M_{n}(I)\right\} \subseteq V\left(\Gamma_{t}\left(M_{n}(R) / M_{n}(I)\right)\right)$.

Note that for $i \neq j, B_{i}+M_{n}(I)=B_{j}+M_{n}(I)$ or $B_{i}+M_{n}(I)$ is not adjacent to $B_{j}+M_{n}(I)$ in $\Gamma_{t}\left(M_{n}(R) / M_{n}(I)\right)$. Since $\left|B_{i}+M_{n}(I)\right|=\left|M_{n}(I)\right|$ we have at least $k+1$ distinct elements in $X$ such that the $k+1$ elements are not adjacent to each other in $\Gamma_{t}\left(M_{n}(R) / M_{n}(I)\right)$,i.e., $\alpha\left(\Gamma_{t}\left(M_{n}(R) / M_{n}(I)\right)\right) \geq k+1$ which is a contradiction. Hence $\alpha\left(\Gamma_{I^{t}}\left(M_{n}(R)\right)\right) \leq k\left|M_{n}(I)\right|$.

2. From Theorem 3.3(2) and (3), we have $\alpha\left(\Gamma_{I^{t}}\left(M_{n}(R)\right)\right) \geq\left|M_{n}(I)\right| \alpha\left(\Gamma_{t}\left(M_{n}(R / I)\right)\right)$. By the previous part, we have $\alpha\left(\Gamma_{I^{t}}\left(M_{n}(R)\right)\right)=\left|M_{n}(I)\right| \alpha\left(\Gamma_{t}\left(M_{n}(R / I)\right)\right)$.

Acknowledgment. This work is supported by the INSPIRE programme (IF 160672) of Department of Science and Technology, Government of India, India for the second author. Further this work is supported by UGC-SAP(DRS-II) programme through the first author.

\section{References}

[1] F.A.A. Almahdi, K. Louartiti, and M. Tamekkante, The trace graph of the matrix ring over a finite commutative ring, Acta Math. Hungar. 156 (1), 132-144, 2018.

[2] D.F. Anderson and P.S. Livingston, The zero divisor graph of a commutative ring, J. Algebra 217, 434-447, 1999.

[3] I. Beck, Coloring of commutative rings, J. Algebra 116, 208-226, 1988.

[4] G. Chartrand, O.R. Oellermann, Applied and algorithmic graph theory, McGraw-Hill, Inc., New York, 1993.

[5] I.N. Herstein, Noncommutative rings, Carus Monographs in Mathematics, Math. Assoc. of America, 1968.

[6] I. Kaplansky, Commutative Rings, University of Chicago Press, Chicago, 1974.

[7] T.Y. Lam, A first course in noncommutative rings, Graduate Texts in Mathematics, Springer-Verlag, New York 2001.

[8] H.R. Maimani, M.R. Pournaki, and S. Yassemi, Zero-divisor graph with respect to an ideal, Comm. Algebra 34 (3), 923-929, 2006.

[9] S.P. Redmond, An ideal-based zero-divisor graph of a commutative ring, Comm. Algebra 31, 4425-4443, 2003.

[10] M. Sivagami and T. Tamizh Chelvam, On the trace graph of matrices, Acta Math. Hungar. 158 (1), 235-250, 2019, https://doi.org/10.1007/s10474-019-00918-5.

[11] D.B. West, Introduction to graph theory, Prentice Hall, 2001. 